\section{§15. Feedback Instability Analysis for Dipole Configuration with lonospheric and Magnetospheric Cavities}

Hiraki, Y. (Nagoya Univ.), Watanabe, T.-H.

Linear eigenmodes of ionospheric feedback instability in the dipole magnetic field $\left(B_{0}\right)$ geometry are investigated by considering the ionospheric and magnetospheric resonant cavities with inhomogeneous Alfvén velocity $\left(v_{\mathrm{A}}\right)$ profiles. The two-field reduced magnetohydrodynamic model ${ }^{1)}$

$$
\begin{aligned}
& \partial_{t} \omega+\boldsymbol{v}_{0} \cdot \nabla_{\perp} \omega=v_{\mathrm{A}}^{2} \nabla_{\|} j_{\|} \\
& \partial_{t} \psi+\boldsymbol{v}_{0} \cdot \nabla_{\perp} \psi+\frac{1}{B_{0}} \nabla_{\|} B_{0} \phi=0
\end{aligned}
$$

is used to describe shear Alfvén wave dynamics, associated with auroral arcs, in a strongly non-uniform magnetic flux tube. Here, $\omega$ is vorticity, $\psi$ is the parallel vector potential, $\phi$ is the scalar potential, and $\boldsymbol{v}_{0}$ is a convection electric drift speed. These equations are coupled with the two-fluid equations in the ionosphere, which is the bottom boundary of the system, to provide the linear dispersion relation for feedback instability,

$$
\left(1-\frac{\sigma}{\Omega}\right) \psi+\alpha \phi=0 .
$$

Here, $\sigma$ is the effective electric drift frequency in the ionosphere, $\alpha$ is the ratio of the Pedersen conductance to the Alfvén impedance, and $\Omega$ is eigenfrequency.

Revisiting a previous study, $\left.{ }^{2}\right)$ linear properties of ionospheric cavity modes (eigenfrequency and eigenfunctions) are examined for a wider parameter range. Figure 1 shows that several high harmonics of these modes are trapped in the cavity region and their magnetic fluctuations $\left(B_{0} \psi\right)$ have a large amplitude at the ionosphere, leading to a high growth rate. By steepening a gradient of $v_{\mathrm{A}}$ on the magnetospheric side, the ionospheric cavity modes are fully diminished as shown in Fig. 2, although the growth rates remain to be small positive values. On the other hand, the lowest fundamental mode has a high growth rate with a large amplitude of magnetic fluctuations at the ionosphere owing to the magnetospheric cavity of $v_{\mathrm{A}}$. Possible spatial and temporal scales of auroral arcs and ULF wave resonances in a realistic situation are discussed in this paper. ${ }^{3)}$

1) Watanabe, T.-H.: Phys. Plasmas 17 (2010) 022904.

2) Lysak, R. L.: J. Geophys. Res. 96 (1991) 1553.

3) Hiraki, Y., and T.-H. Watanabe: J. Geophys. Res. (2011) submitted.
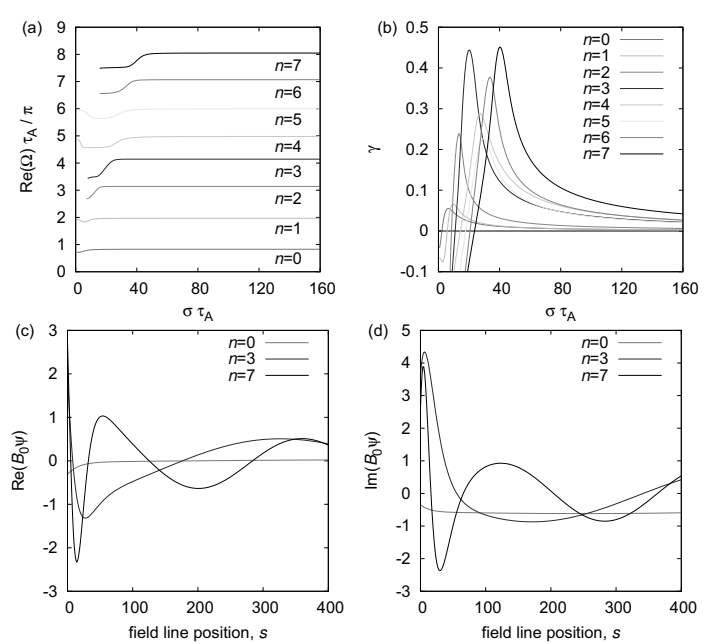

Fig. 1: Eigenfrequency $\Omega$ and eigenfunction $B_{0} \psi$ of shear Alfvén modes obtained by equation (3) with the impedance ratio $\alpha=5$ and the ionospheric cavity of $v_{\mathrm{A}}$. Panels (a) and (b) show real and imaginary parts of $\Omega$, normalized by the Alfvén transit time $\tau_{\mathrm{A}}$ as a function of the ionospheric electric drift frequency $\sigma$, for lower eight harmonics $n=0-7 ; \gamma=\operatorname{Im}(\Omega) \tau_{\mathrm{A}} / \pi$. Field-line distribution of (c) real and (d) imaginary parts of $B_{0} \psi$ for the maximum $\gamma$ of harmonics $n=0,3$, and 7 .
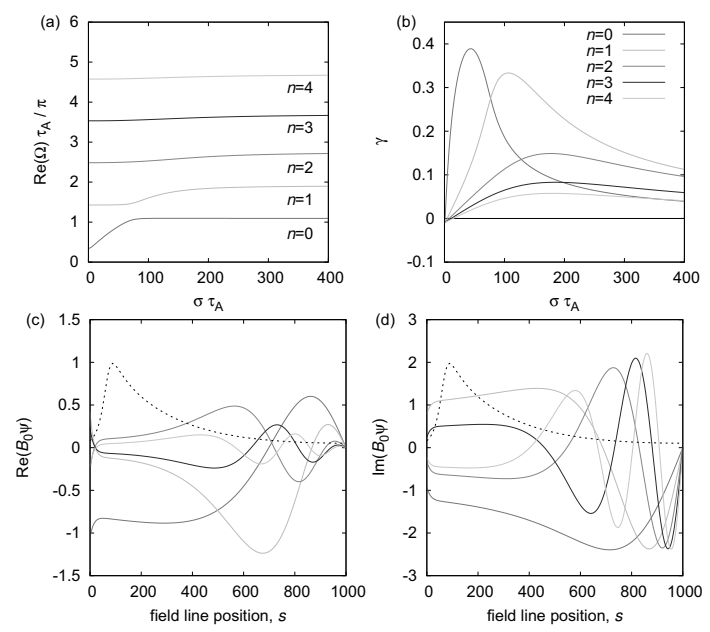

Fig. 2: (a) Real and (b) imaginary parts of normalized $\Omega(\sigma)$ obtained by equation (3) with $\alpha=5$ and the ionospheric and magnetospheric cavities of $v_{\mathrm{A}}$ (dotted lines in (c) and (d)). The growth rate for a harmonic $n=0$ recovers whereas the rates for ionospheric cavity modes become quite small. Field-line distribution of (c) real and (d) imaginary parts of $B_{0} \psi$ for the maximum $\gamma$ of harmonics $n=0-4$. Amplitudes for $n=0$ are highest at $s=0$. 